\title{
Non-Destructive Robotic Assessment of Mango Ripeness via Multi-Point Soft Haptics
}

\author{
Luca Scimeca $^{1}$, Perla Maiolino ${ }^{1}$, Daniel Cardin-Catalan ${ }^{2}$, \\ Angel P. del Pobil ${ }^{2}$, Antonio Morales ${ }^{2}$ and Fumiya Iida ${ }^{1}$
}

\begin{abstract}
To match the ever increasing standards of fresh products, and the need to reduce waste, we devise an alternative to the destructive and highly variable fruit ripeness estimation by a penetrometer. We propose a fully automatic method to assess the ripeness of mango which is non-destructive, allows the user to test multiple surface areas with a single touch and is capable of dissociating between ripe and non-ripe fruits. A custom-made gripper equipped with a capacitive tactile sensor array is used to palpate the fruit. The ripeness is estimated as mango stiffness extracted through a simplified spring model. We test the framework on a set of 25 mangoes of the Keitt variety, and compare the results to penetrometer measurements. We show it is possible to correctly classify $88 \%$ of the mango without removing the skin of the fruit. The method can be a valuable substitute for non-destructive fruit ripeness testing. To the authors knowledge, this is the first robotics ripeness estimation system based on capacitive tactile sensing technology.
\end{abstract}

\section{INTRODUCTION}

With ever increasing demand for high quality horticultural products, and an increase in their acceptable marketing standards, there is a need to find fast, reliable and autonomous processes which can provide these guarantees [1]. The quality of crop is dependent on several pre-harvest factors, among which weather conditions, growing land, irrigation patters, chemical treatments and others still [2]. After harvesting, an important characteristic determining quality, and which has a direct impact in the marketability of the produce, is ripeness [3]. Besides appropriate harvesting time, determining the ripeness of horticultural produce is useful for classification, transportation, handling and the security of its quality.

In crop such as bananas, strawberry, watermelons and tomato, visual cues have been shown to be sufficient in assessing ripeness and classifying produce accordingly [4][6]. Other fruits like kiwis, blueberry or mango, however, do not provide useful visual diversity between ripe and unripe units. In this paper we focus on ripeness assessment of mango, a high value agricultural and food product, which shows different ripeness visual cues depending on its variety. Given the large variety of mangoes (over a 1000 only in India), assessing ripeness by machine vision is discouraged.

The current approach for ripeness assessment of mango and similar fruits is by measuring their firmness through

This work was funded by the UK Agriculture and Horticulture Development Board (CP 172), Physical Sciences Research Council (EPSRC) MOTION grant [EP/N03211X/2] and Ministerio de Economía (DPI201569041-R).

1 The authors are with the Bio-Inspired Robotics Lab. Cambridge University Dept. of Engineering, Trumpington St Cambridge CB2 1PZ, UK

2 The authors are with the Robotic Intelligence Laboratory, Universitat Jaume I, Av. Vicent Sos Baynat s/n, 12071 Castellón, Spain.

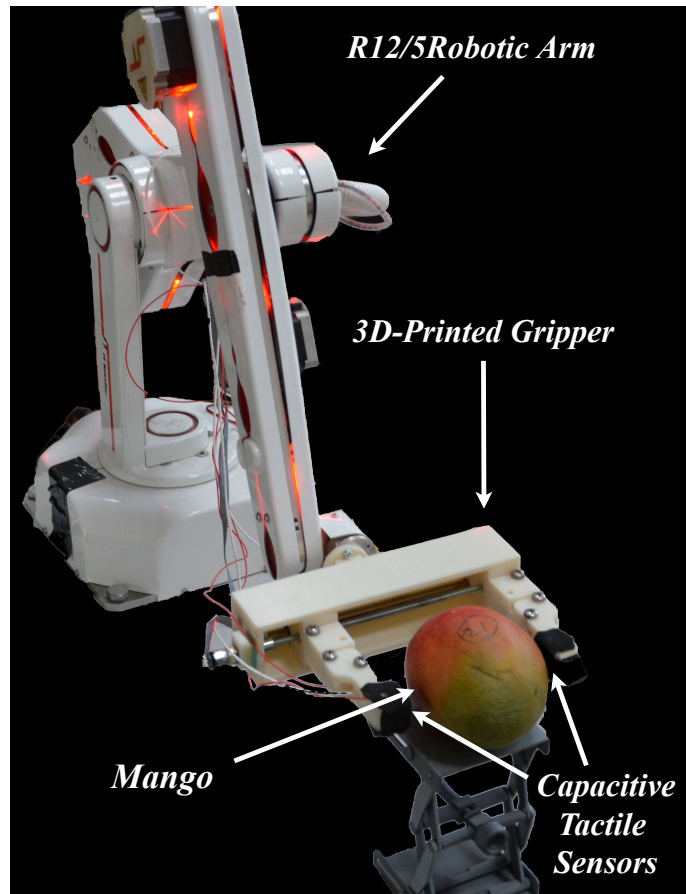

Fig. 1: Mango ripeness assessment robotic setup.

a penetrometer instrument [7], [8]. A penetrometer is a pressure tester with a cylindrical head, which is usually inserted in the pulp of test fruit at a set depth, and a speed approximately controlled by an operator. The firmness values of fruit tested by a penetrometer may largely vary depending on the instrument's user [9]. Moreover, penetrometer testing is a destructive procedure, as the post-measurement fruit damage is irreversible, and the tested fruit must therefore be discarded. Finally, the firmness distribution of the pulp of fruit across its surface may largely vary, and only successive single penetrometer tests in different locations can insure a thorough firmness assessment.

In this paper, we propose a method for testing the ripeness of mango by means of touch. The proposed method is nondestructive, and allows the user to test multiple surface areas with a single touch. The method designed is possible given the use of capacitive tactile sensing technology, endowing end-effectors with the ability to retrieve multiple contact pressure readings in relatively small areas. We make use of a custom-made probe equipped with a capacitive tactile sensor array in order to palpate the fruit and thereby assess its ripeness. We model the mango's pulp and skin behavior when deformed through a simple spring system and thus 


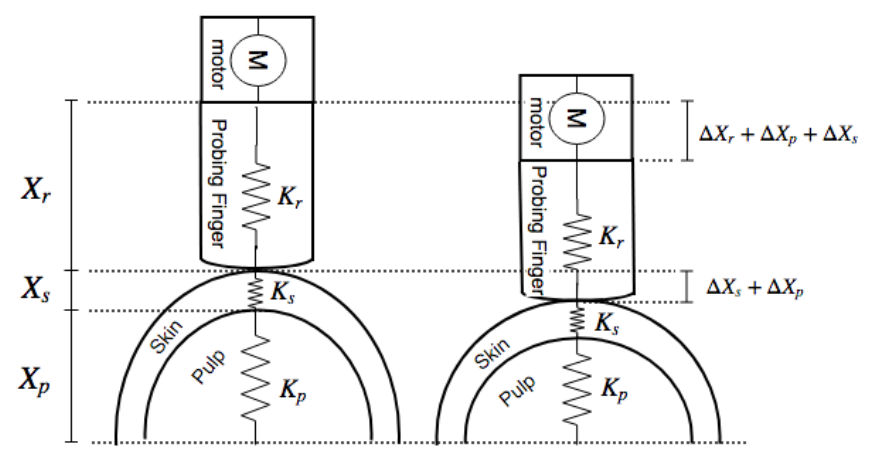

Fig. 2: Stiffness model.

retrieve fruit firmness as a stiffness measure.

This paper is structured as follows. Section I-A presents a brief review of the current technologies available for ripeness assessment of horticultural produce. In Section II the methods are explained, including the theoretical framework for ripeness assessment. In Section II-A, the gripper design in Section II-B, and the tactile sensor technology used in Section II-C. In Section III the results are reported and finally a conclusion in given in Section IV.

\section{A. Approaches for ripeness Assessment}

In the past few decades, with advancements of machine learning and vision, systems have been made showing it is possible to assess the ripeness of horticultural produce by visual cues [4]-[6]. These systems, however, are limited to produce which show differences in chromacity at various ripeness stages. For mangoes specifically, systems using machine vision have previously been explored [10]. Given the large variety of mango types, however, these solutions were only limited to specific families.

Recently, spectral techniques have been used to assess the quality of post-harvested produce [11]-[13]. More specifically, Raman imaging, Fluorescence imaging, Laser backscattering imaging, Hyperspectral imaging and Nuclear magnetic techniques have been shown to be able to classify produce lacking chromaticity differences into various stages of ripeness. The equipment required for said methods, however, is usually bulky and the information processing often computationally intensive, making it hard to create solutions which can be exported in the field, or do not require the transportation of produce to appropriately equipped areas.

When assessing ripeness, consumers use a combination of tactile sensing and visual cues. The physical probing of produce is indeed one of the oldest modalities for ripeness assessment, and brought the advent of penetrometer measurements [7], non the least because of the instrument's ease of use and transportability. Recent years have seen a rapid development of robotics technology in the context of agriculture, mainly related to transport and harvesting [14][17]. Moreover, technological advances in tactile sensing and perception [18], [19] have changed the landscape for tactile based inference procedures [20], [21]. In this context, however, robotics solutions for post-harvest quality assessment remain a largely unexplored area.

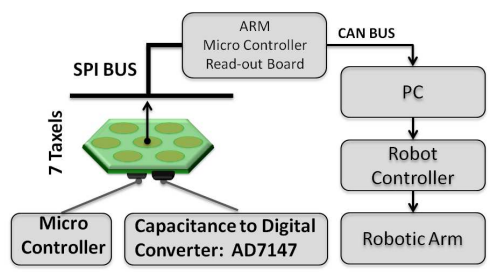

(a)

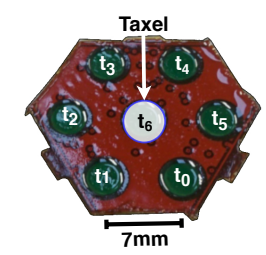

(b)
Fig. 3: (a) The CySkin technology architecture. (b) The CySkin patch used for the experiments.

\section{METHODS}

\section{A. Stiffness Model}

We propose a palpation procedure to assess the ripeness of mango, and devise a simplified system to model the fruit's pulp and skin behavior throughout palpation. In the model we exemplify the scenario where a finger, equipped with a force sensor, is in contact with the surface of a mango. The finger is actuated by a motor, and its displacement is known by means of a motor encoder. Fig. 2 shows the modeled elastic response of the probing finger, and the object's surface, as system of springs. We choose a linear model as the simplest mechanical model of the fruit, and make the simplifying assumption that each spring is constant. The probing finger has a spring stiffness constant of $K_{r}$, while the mango can be seen as a two layered structure, the first layer of which consists of the spring response of the skin, with a spring constant of $K_{s}$, and the second the spring response of the pulp, with a spring constant of $K_{p}$. The lengths of each are also respectively $X_{r}, X_{s}$ and $X_{p}$. The estimation of the produce's stiffness is equivalent to retrieving the elastic constant $K_{e}$. The motor generates a torque capable of directly influencing the distance between the finger and the produce. At equilibrium, the forces generated by the probing fingers $F_{r}$ equate the reacting forces from the produce's surface $F_{e}$, thus $F_{r}=F_{e}$, i.e.:

$$
F_{r}=\left(\frac{1}{K_{s}}+\frac{1}{K_{p}}\right)^{1}\left(\Delta X_{p}+\Delta X_{s}\right) .
$$

As the skin of mango is much stiffer than its pulp, when applying a small displacement though the motor, it is useful to make the simplifying assumption that $K_{s} \approx \infty$, therefore $\Delta X s=0$ and

$$
F_{e}=K_{p} X_{p} .
$$

Finally, the motor displacement as computed by the encoder corresponds to $\Delta X_{m}=\Delta X_{r}+\Delta X_{p}$. So from equation 2 , and the simplifying assumption we have:

$$
\begin{aligned}
& K_{e}(1+\epsilon)=\frac{F_{r}}{\Delta X_{r}+\Delta X_{p}} \\
& \text { where, } \epsilon=\frac{\Delta X r}{\Delta X_{r}+\Delta X_{p}}
\end{aligned}
$$

i.e. when the motor displacement is large and/or the compression of the sensor $\Delta X_{r}$ is much smaller than that of the mango $\Delta X_{p}, \epsilon$ can be neglected. 


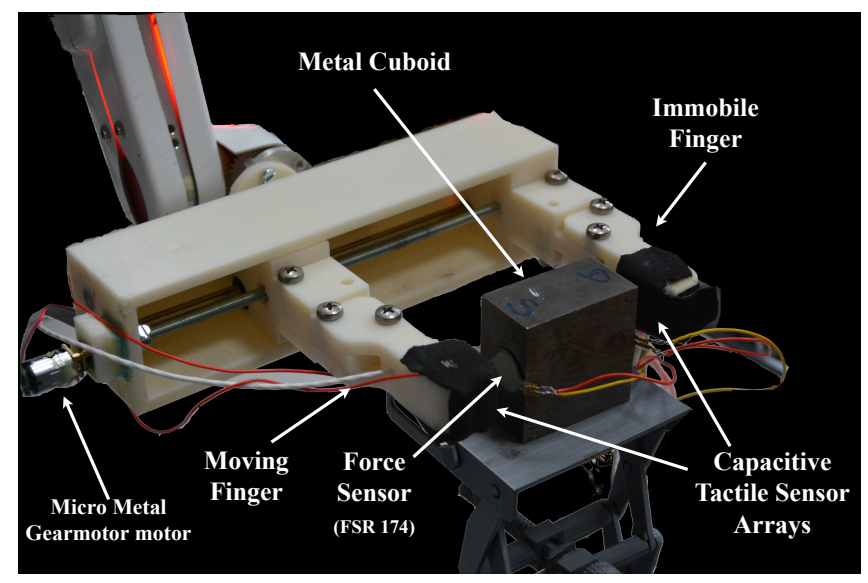

Fig. 4: Gripper, and force calibration set-up.

\section{B. Probing Gripper Mechanism}

Fig. 4 shows the gripper used for the experiments. The gripper is composed by a main rectangular case containing a lead screw and two metallic rods. The chamber contains two opposite fingers, which remain parallel to each other throughout the gripper's actuation. We designed two fingers with flat surfaces at the extremities, capable of holding the referenced tactile sensor. A central actuation unit reduces the distance between the fingers by actuating one finger thorough a Micro Metal Gearmotor motor, with a 6:1 gear ratio and equipped with a rotary encoder. The rotational actuation movement is then transfered into a linear displacement by the lead screw and metallic rod. We control the motor via a TB6612FNG Dual Motor Driver Carrier controller. Each gripper component was 3D-printed, for fast prototyping.

\section{Tactile Sensor Technology and Data Acquisition}

The tactile sensor (CySkin) used for the experiments is described in [22]. The adopted sensing mode is based on the capacitive transduction principle. A capacitive transducer (i.e., a tactile element, or taxel) is organized in a layered structure: the lower layer consists of the positive electrode, which is mounted on a Flexible Printed Circuit Board (FPCB). The dielectric for the sensor is here fundamental. The deformation of a too soft dielectric layer, like air, may reach its saturation before inducing any deformation in the pulp of a mango. From equation 4 it is clear how the deformation of the mango surface must be grater than that of the sensor, for the assumptions to hold. Knowing typical mango firmness ranges ${ }^{4}$, we choose a 3D-printed dielectric layer of $2 \mathrm{~mm}$, composed of VeroBlack rubber with A-27 Shore coefficient.

In the experiments we use 2 hexagonal shaped modules, each placed in the inner flat extremity of a finger in the gripper (Fig. 4). Each module hosts 6 taxels (Fig. 3b), as well as the Capacitance to Digital Converter (CDC) chip (namely, the AD7147 from Analog Devices) for converting capacitance values to digital. The CDC chip can measure

\footnotetext{
${ }^{4}$ https://www.mango.org/wp-content/uploads/2017/10/Mango_Maturity _And_Ripeness_Guide.pdf
}



Fig. 5: Flowchart of the touch experiment for a mango.

variations in capacitance values with 16 bits of resolution. All the modules are interconnected and communicate through an SPI bus to a read-out board which performs a preliminary processing of the tactile sensor data and send them to the PC through CAN bus (Fig. 3a) with a sensitivity of $0.32 \mathrm{pF}$. In this context, the normal forces exerted on the sensor produce variations in capacitance values reflecting the varied pressure over the taxel positions. A sensor reading, or tactile image, from the tactile sensors described is produced at $20 \mathrm{~Hz}$, and corresponds to two 6-dimensional arrays, where each element contains the capacitance variation value of the corresponding taxel within each finger in the gripper. Here and for the remainder of the experiments, each taxel is considered a separate tactile sensing unit.

\section{Robot and Experimental Set-Up}

To perform the mango experiments the end-effector, coupled with the tactile sensor, was mounted onto an STRobotics R12/5 $\mathrm{arm}^{3}$. The R12/5 robotic arm was controlled open-loop in Cartesian coordinates. A teach-pendant was used to manually teach the robot the starting position with the arm and end-effector facing forward (Fig. 1).

After the robot arm reaches the starting position, and a test produce is placed within the end-effector's reach, the probing experiment consists of three stages: a reaching, a probing and a release stage (Fig. 5).

In the first phase, the gripper's moving finger is driven towards its immobile counterpart at $1 \mathrm{~mm} / \mathrm{s}$. When any taxel, in both fingers, reads values above $5 \%$ of their maximum calibration, a touch is detected and the gripper is stopped.

In the second phase, the mobile finger is further actuated to close the gripper until either of two conditions are met: first, the last touching taxel has moved of at least $1 \mathrm{~mm}$ into the flesh of the fruit; second, the encoders do not change value over two consecutive readings (i.e. the motor is at equilibrium at its maximal gripping force).The slow motion induce quasi-static interaction validating the model's

${ }^{3}$ http://www.robotshop.com/uk/st-robotics-r12-5-axis-articulated-robotarm.html 


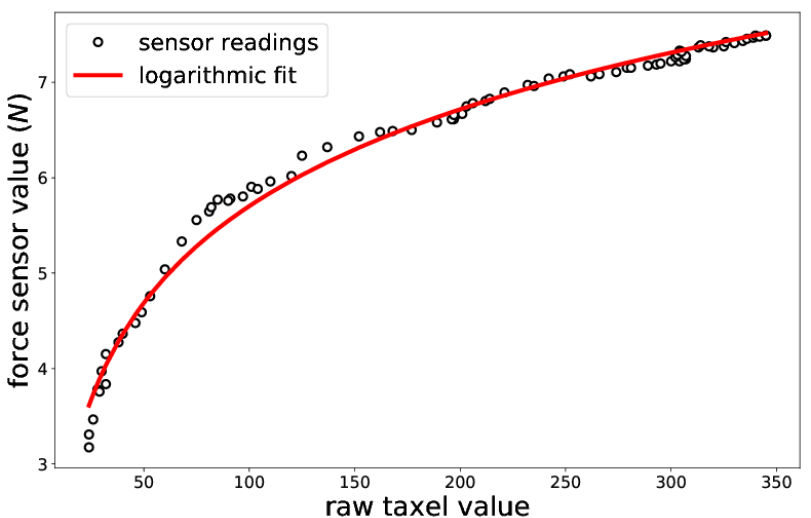

Fig. 6: Raw taxel value to force logarithmic fit on taxel 3.

static assumptions. The depth of $1 \mathrm{~mm}$ was chosen to induce enough deformation in the mango, while working within the linear range of the sensor (Fig. 6, force range $[0,5.5] N$ ).

In the third phase, the gripper mobile finger is reversed at $1 \mathrm{~mm} / \mathrm{s}$ to the gripper's fully opened state (Fig. 5).

The system has been implemented in MATLAB, synchronizing the gripper control and the sensors acquisition. The tactile images are thus recorded at $\approx 15 \mathrm{~Hz}$ throughout the run of the second stage and later used to retrieve the stiffness of the touched produce.

\section{RESULTS}

\section{A. Sensor Force Calibration}

To make use of the theoretical spring model we converted the tactile sensor pressure response, of each taxel, in force. To achieve accurate conversions, the end-effector coupled with the tactile sensor was made to close onto a stiff metal cuboidal object. The opposite surface areas of the cuboid, in contact with the end-effector's fingers, were covered by two force sensitive resistors FSR 174 sensors, previously calibrated to measure forces in $0-10 N$ range with an accuracy of $0.01 N$ (Fig. 4). In the experiment, the gripper was actuated to close at $1 \mathrm{~mm} / \mathrm{s}$ until motor torque limit. The force sensor response and corresponding taxel values were sampled at $\approx 15 \mathrm{~Hz}$. Given prior knowledge of the dielectric layer deformation behavior [?], we fit a logarithmic curve of the form $f(x)=a \log (b x-1)+c$, mapping the pressure response to the punctual forces registered during the calibration. The $a, b$, and $c$ parameters were optimized by least squares. Fig. 6 shows an example force fit for taxel 3, all other taxels were similarly calibrated.

\section{B. Mango Penetrometer Testing}

A set of 25 mangoes of the Keitt variety were used for testing. The mango were divided in three subsets and made to ripen at room temperature for 1,3 and 5 days, increasing the ripeness differences amongst subsets (Fig. 7a).

We use a penetrometer instrument to retrieve ground truth mango firmness measurements. Penetrometer tests were done following industrial standards. The skin of each mango was removed before the measurement, and pressure was

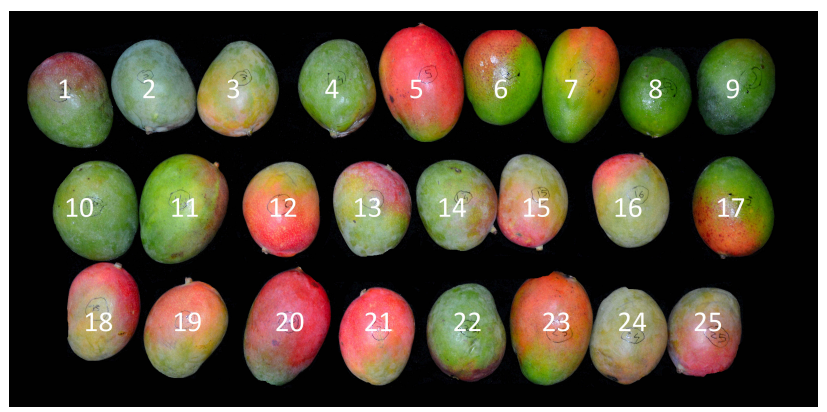

(a)

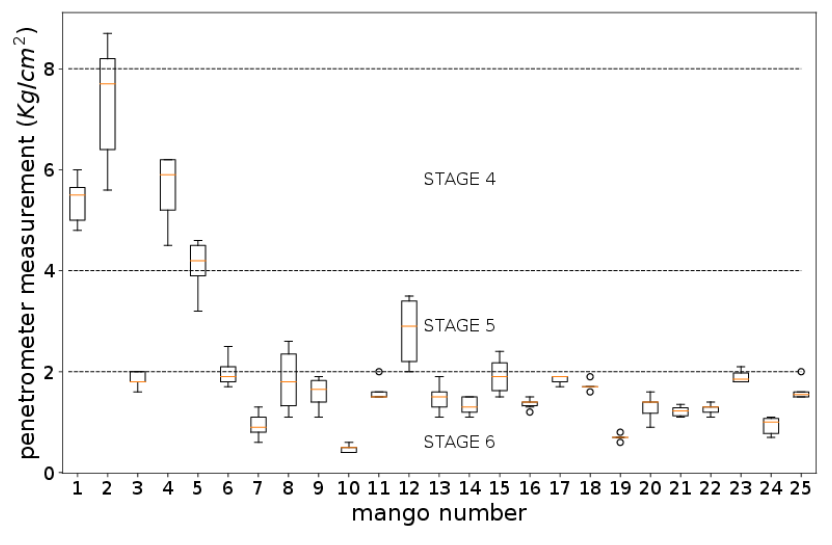

(b)

Fig. 7: (a) Mangoes used for the experiments at purchase time. (b) Penetrometer measurements of each mango when tested at a distance of 1, 3 and 5 days from purchase.

applied to reach the penetrometer's head full insertion in approximately $2 s$. A total of 10 measurements were done on each mango. Fig. $7 \mathrm{~b}$ shows the penetrometer measurement values for each fruit in the test set. Mangoes 1-9 were tested on day one, mangoes 10-18 were tested on day two and mangoes 19-25 were tested on day five. Comparing the measured values to standard ripeness levels ${ }^{4} 20$ mangoes were found to be at ripeness stage 6 (very ripe), 2 mangoes at ripeness stage 5 (ripe) and 3 mangoes at ripeness stage 4 (non-ripe). Fig. 7b shows how penetrometer tests, even when the instrument is operated by the same user, are somewhat variable (average standard deviation of penetrometer measures $\approx 0.287 \mathrm{Kg} / \mathrm{cm}^{2}$ ), and do not always clearly collocate a mango in a ripeness stage.

\section{Stiffness Measurement Analysis}

We analyze whether from the raw tactile information is possible to dissociate between ripe and unripe mangoes. Fig. 8 shows the raw tactile sensor response, when touching fruits at ripeness stage 4 , and 6 (as determined by penetrometer testing). In Fig. 8a it is clear how the ripeness stage information is captured by the sensor response. Moreover, we observe how different taxels activate at different times and with different intensity depending on the mango. The variability is mainly due to the curvature of the fruit against the sensor's flat surface. 


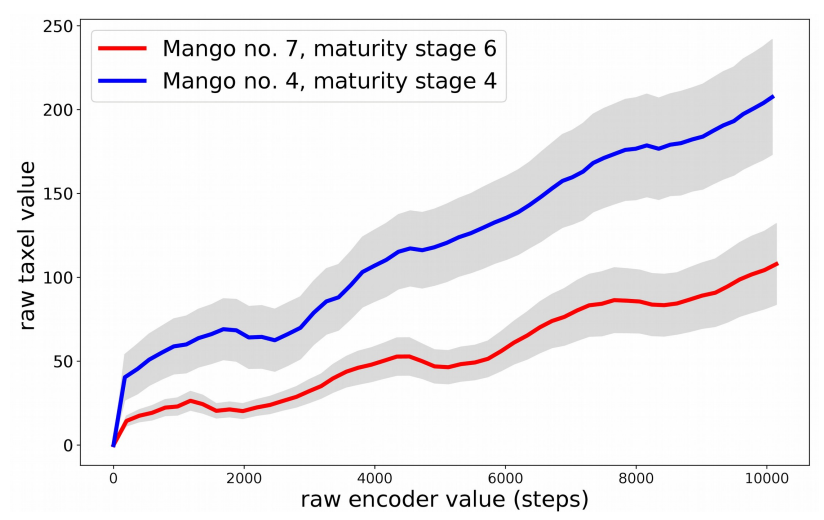

(a)

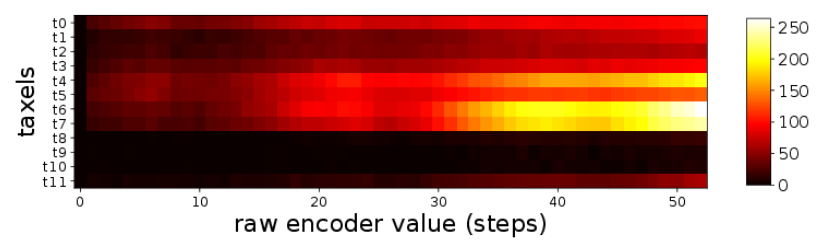

(b) Mango no. 7 , ripeness stage 6 .

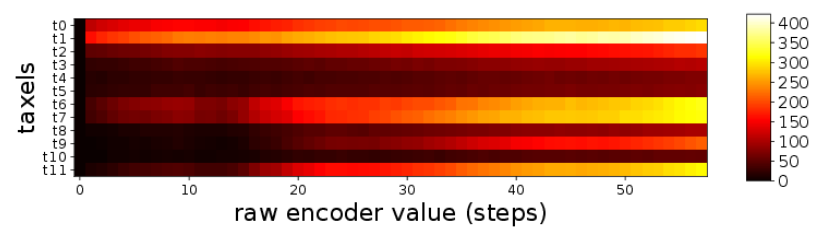

(c) Mango no. 4, ripeness stage 4 .

Fig. 8: (a) Mean and error of all raw tactile sensing units, (b) raw tactile sensor response, when performing the touching experiment on mango number 7 , at ripeness stage 6 , and (c) a mango number 4 , at ripeness stage 4.

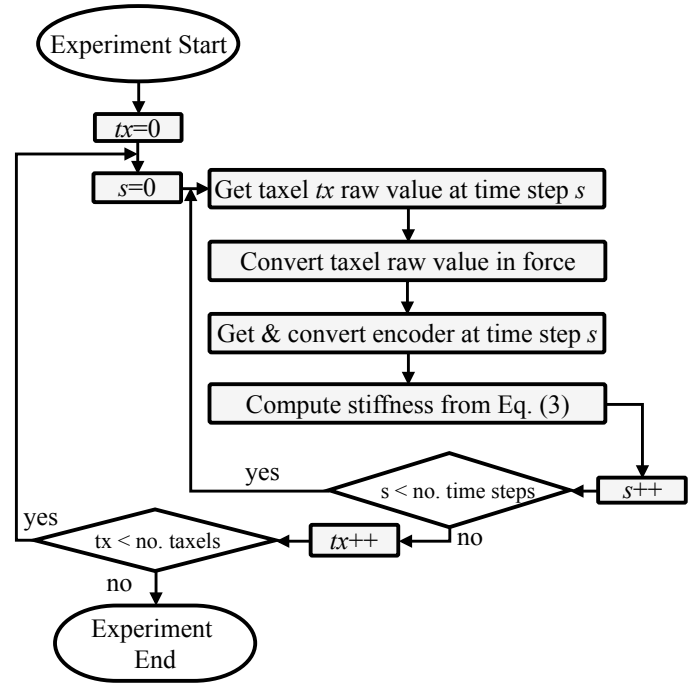

Fig. 9: Flowchart of the stiffness extraction processing.

During the experiments with each sample, the recorded sequential sensor response was used, together with equation 3 , to retrieve the stiffness $K_{e}$ of the mango's pulp (Fig. 9) We compare the computed stiffness of each mango against average penetrometer measurements in two scenarios: one, as an average of the stiffnesses computed by all sensing units (Fig. 10a), and two, as an average of the four taxels registering the highest change in pressure over the course of each touch experiment (Fig. 10b). Fig. 10 shows how it is only possible for the sensor (y-axis in figure) to separate between ripe and non-ripe mangoes when given the opportunity to choose reliable tactile sensing units, depending on the touch experiment. The result emphasizes the need of sensors capable of drawing multiple measurements at once, as any one measurement might be unreliable in its ripeness estimation. Moreover, the sparse sensor response for mango at ripeness stage 6 (Fig. 10b), suggests that the skin of mangoes has a non-linear influence on the measured pressures. Given typical mango skin thicknesses, such influence is negligible for nonripe mangoes, where the stiffness of the pulp dominates the sensor readings. For ripe and very ripe mangoes, however, the stiffness of the skin, at times, induces the stiffness of the whole fruit to be much higher than its pulp.

For the final estimation of mango stiffness after contact, we thus limit the computation on an average of the four taxels measuring the highest change in pressure over the course of the touch experiment's second stage. In this context, the use of multiple taxels for stiffness estimations allows for the dismissal of outliers generated by the curvature of the fruit. We consider non-ripe the mangoes whose stiffnesses is in the range $K_{e}>9.7$, and ripe the mangoes those where $K_{e}<9.7$. The ripeness threshold was chosen to maximize accuracy over the tested fruit. Table I reports the thresholding results. In particular, we find we can classify $88 \%$ of the tested fruit correctly, and accurately detect all the non-ripe samples in the tested mangoes.

\section{CONCLUSIONS}

Given the lack of standard, non destructive and nonchemical tests for assessing the ripeness of fruit, we devise a ripeness testing method based on capacitive tactile sensing technology. We devise a custom made gripper, supplied with 12 capacitive tactile sensing units distributed homogeneously over two fingertips. We perform experiments by which the gripper close onto the flesh of test fruit until a pre-set depth is reached, while recording tactile image sequences. The tactile image sequences, together with a spring stiffness model, are used to retrieve the stiffness of the palpated fruit and assess its ripeness. We test the proposed method on a set of 25 mango fruit of the Keitt variety. We compare the whole fruit stiffness computations to pulp ripeness measurements based on a standard penetrometer instrument. Results show that the tactile based ripeness assessment method is capable of classifying mangoes into ripe or non-ripe, with accuracies increasing with the stiffness of the pulp. Moreover, since the proposed method, based on capacitive tactile technology, hinges on the relationship between flesh stiffness and ripeness of target produce, we argue the method is valid for other types of horticultural produce showing such 


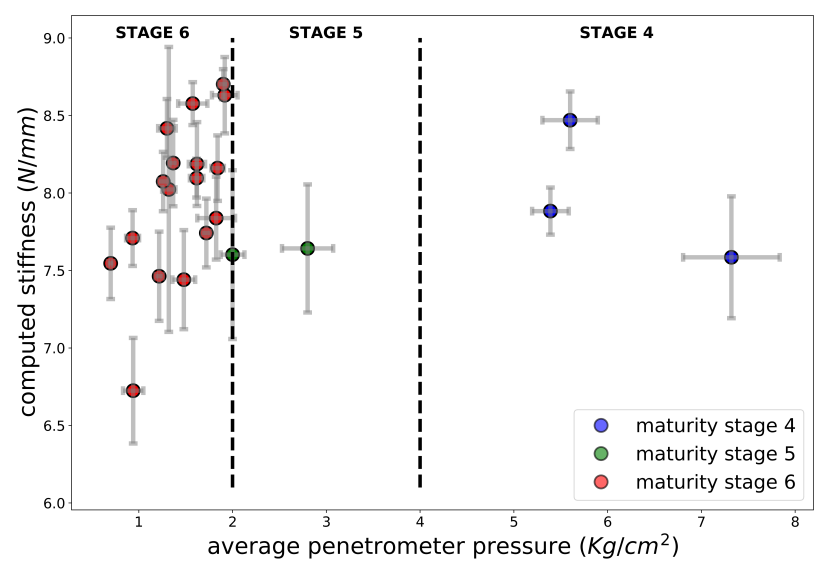

(a)



(b)

Fig. 10: (a) Computed stiffness averages from all sensing units and (b) stiffness averages of the 4 taxels recording the highest change in pressure throughout the experiment, against average penetrometer measurements. Each point in the plot is a different mango in the dataset.

relationship, e.g. tomatoes, grapes, apricot, cherries, kiwis and others besides, some of which may present difficulties for visual based ripeness assessment.

A non-linear effect to the stiffness estimation, due to the intact skin of the mango, was observed. The effect makes the distinction between stage 5 and 6 mangoes hard, in general. Future work should allow the differentiation between skin and pulp stiffness, increasing the comparability of the results with standard measurements.

Finally, as the scale of penetrometer measurements may vary depending on the user, the proposed method presents clear transferability advantages for testing ripe over nonripe mangoes. Moreover, the method is non-destructive, the sensor technology utilized can test several surface locations at once, the gripping technology is light thus can be mobile, and its usage does not require any specialized expertise.

\section{REFERENCES}

[1] A. A. Kader and R. S. Rolle, The role of post-harvest management in assuring the quality and safety of horticultural produce. Food \& Agriculture Org., 2004, vol. 152.
TABLE I: Results Summary

\begin{tabular}{l|llll}
\hline STAGE & No. & Correctly classified & Misclassified & Accuracy(\%) \\
\hline 5\&6 (ripe) & 22 & 19 & 3 & 86 \\
4 (non-ripe) & 3 & 3 & 0 & 100 \\
\hline \multicolumn{7}{r}{}
\end{tabular}

[2] C. E. Sams, "Preharvest factors affecting postharvest texture," Postharvest Bio. and Technology, vol. 15, no. 3, pp. 249-254, 1999.

[3] A. A. Kader, "Fruit maturity, ripening, and quality relationships," in Int. Symp. Effect of Pre-\& Postharvest factors in Fruit Storage 485, 1997, pp. 203-208.

[4] N. B. A. Mustafa, N. A. Fuad, S. K. Ahmed, A. A. Z. Abidin, Z. Ali, W. B. Yit, and Z. A. M. Sharrif, "Image processing of an agriculture produce: Determination of size and ripeness of a banana," in Inf. Technology, 2008. ITSim 2008. Int. Symp. on, vol. 1. IEEE, 2008, pp. 1-7.

[5] M. S. S. Rizam, A. F. Yasmin, M. A. Ihsan, and K. Shazana, "Nondestructive watermelon ripeness determination using image processing and artificial neural network (ann)," World Academy of Sci., Eng. and Technology, vol. 38, pp. 542-546, 2009.

[6] E. El Hariri, N. El-Bendary, A. E. Hassanien, and A. Badr, "Automated ripeness assessment system of tomatoes using pca and svm techniques," in Computer Vision and Image Processing in Intelligent Syst. and Multimedia Technologies. IGI global, 2014, pp. 101-130.

[7] G. Blanpied, W. Bramlage, D. Dewey, R. LaBelle, L. Massey Jr, G. Mattus, W. Stiles, and A. Watada, "A standardized method for collecting apple pressure test data," 1978.

[8] J. A. Abbott, "Quality measurement of fruits and vegetables," Postharvest Biology and Technology, vol. 15, no. 3, pp. $207-225,1999$.

[9] M. C. Bourne, "Fruit texture-an overview of trends and problems," J. of Texture Studies, vol. 10, no. 1, pp. 83-94, 1979.

[10] V. E. Nambi, K. Thangavel, and D. M. Jesudas, "Scientific classification of ripening period and development of colour grade chart for indian mangoes (mangifera indica 1.) using multivariate cluster analysis," Scientia Horticulturae, vol. 193, pp. 90-98, 2015.

[11] D. Slaughter, "Nondestructive maturity assessment methods for mango," University of California, Davis, pp. 1-18, 2009.

[12] S. Jha, K. Narsaiah, A. Sharma, M. Singh, S. Bansal, and R. Kumar, "Quality parameters of mango and potential of non-destructive techniques for their measurement-a review," J. of Food Sci. and Technology, vol. 47, no. 1, pp. 1-14, 2010.

[13] A. Hussain, H. Pu, and D.-W. Sun, "Innovative nondestructive imaging techniques for ripening and maturity of fruits-a review of recent applications," Trends in Food Sci. \& Technology, 2017.

[14] S. Hayashi, K. Ganno, Y. Ishii, and I. Tanaka, "Robotic harvesting system for eggplants," Japan Agricultural Research Quarterly: JARQ, vol. 36 , no. 3, pp. 163-168, 2002.

[15] T. T. Nguyen, K. Vandevoorde, E. Kayacan, J. De Baerdemaeker, and W. Saeys, "Apple detection algorithm for robotic harvesting using a rgb-d camera," in Int. Conf. of Agricultural Eng., Zurich, Switzerland, 2014.

[16] Q. Feng, W. Zou, P. Fan, C. Zhang, and X. Wang, "Design and test of robotic harvesting system for cherry tomato," Int. J. of Agricultural and Bio. Eng., vol. 11, pp. 96-100, 2018.

[17] J. Hughes, L. Scimeca, I. Ifrim, P. Maiolino, and F. Iida, "Achieving robotically peeled lettuce," IEEE Trans. Robot., 2018.

[18] N. Herzig, P. Maiolino, F. Iida, and T. Nanayakkara, "A variable stiffness robotic probe for soft tissue palpation," IEEE Trans. Robot., vol. 3, no. 2, pp. 1168-1175, 2018.

[19] U. Culha, S. G. Nurzaman, F. Clemens, and F. Iida, "Svas3: strain vector aided sensorization of soft structures," Sensors, vol. 14, no. 7, pp. 12 748-12 770, 2014.

[20] R. Pfeifer, F. Iida, and M. Lungarella, "Cognition from the bottom up: on biological inspiration, body morphology, and soft materials," Trends in cognitive sciences, vol. 18, no. 8, pp. 404-413, 2014.

[21] L. Scimeca, P. Maiolino, and F. Iida, "Soft morphological processing of tactile stimuli for autonomous category formation," in 2018 IEEE International Conference on Soft Robotics (RoboSoft). IEEE, 2018.

[22] A. Schmitz, P. Maiolino, M. Maggiali, L. Natale, G. Cannata, and G. Metta, "Methods and Technologies for the Implementation of Large-Scale Robot Tactile Sensors," IEEE Trans. on Robotics, vol. 27, no. 3, pp. 389-400, June 2011. 